\title{
Defensive Sniping and Efficiency in Simultaneous Hard-Close Proxy Auctions*
}

\author{
Greg Taylor ${ }^{\dagger}$ \\ Oxford Internet Institute, University of Oxford, Oxford, UK
}

23rd September 2011

\begin{abstract}
A well-known myopic bidding strategy fails to support an equilibrium of simultaneous ascending proxy auctions for heterogeneous items when a hard-close rule is in place. This is because, in common with the single-auction case, last minute bidding (sniping) is a best response to naive behaviour. However, a modification to the myopic strategy in which all bidders submit an additional bid in the closing stages of the auction-a practice I call 'defensive sniping'-is shown to yield an efficient, belief-free equilibrium of such environments. This equilibrium is essentially unique within the class of belief-free, efficient equilibria.
\end{abstract}

Keywords: hard-close; simultaneous proxy auctions; efficiency; myopic bidding; belieffree

JEL classifications: D44; C78

\section{This article was published as as:}

TAYLOR, G. (2012): "Defensive Sniping and Efficiency in Simultaneous Hard-Close Proxy Auctions," Journal of Mathematical Economics, 48(1), 51-58.

doi: $10.1016 /$ j.jmateco.2011.11.006.

\footnotetext{
*This and other papers are available at http://www.greg-taylor.co.uk. I am especially grateful to Robin Mason for many useful discussions. Maksymillian Kwiek, David Myatt, and an annonymous referee also provided invaluable comments and suggestions that have materially enriched this paper. I thank participants at the 63rd European Meeting of the Econometric Society (Univesità Bocconi, Milan) and various seminars for their feedback on an earlier draft of this manuscript. The Economic and Social Research Council provided generous financial support for this project.

${ }^{\dagger}$ Correspondence address: Oxford Internet Institute, University of Oxford, 1 St. Giles, Oxford, UK, OX1 3JS. Email: greg.taylor@oii.ox.ac.uk.

${ }^{\ddagger}$ Journal pagination is indicated in the margins.
} 


\section{INTRODUCTION}

This paper is concerned with the question of how bidders can coordinate in a process of price discovery, whilst still discouraging opportunistic behaviour in hard-close simultaneous ascending proxy auctions. ${ }^{1}$ This kind of auction has been popularised by Internet auction site eBay, which uses such auctions to concurrently sell millions of items at any given time. Hard-close proxy auctions have an obvious appeal for selling goods onlineallowing a decentralised and asynchronous bidding process, whilst guaranteeing a timely end to the auction. There are also other contexts in which a fixed end time might be appealing such as when some bidders have a strategic incentive to prolong the auction, when the items for sale are perishable, or when uncertainty over the ending time causes complementary investments to be delayed.

Auctions that have a hard-close rule often induce bidders to bid very close to the ending time (a practice known as sniping), ${ }^{2}$ which has been linked to a general failure to attain efficiency. ${ }^{3}$ Given the increasingly prominent role of this kind of auction in consumer-to-consumer and business-to-consumer electronic commerce, it is therefore important to understand if and how the efficiency problem can be remedied. One way to mitigate this problem in the case of a single-item auction is to introduce a proxy bidding system that submits incremental bids up to some reservation price on the bidder's behalf: provided that all bidders submit a proxy bid equal to their true value at the start of the auction, no bidder can profitably snipe and the result will be the efficient allocation. ${ }^{4}$ The proxy solution is, however, problematic in the case that there are multiple, heterogeneous items for sale. Bidders then face a trade-off between winning the most desirable item (the one to which they attribute the highest value) and winning the cheapest one. Moreover, bidders will not generally agree on the relative desirability of the items. Unlike the bidding problem in the single-auction case, a simultaneous heterogeneous good auction therefore presents a non-trivial coordination problem and large premature proxy bids risk leaving bidders committed to winning items that subsequently turn out to be sub-optimal (in the sense that there are alternative items that offer a better value-price margin). Moreover, attempts to hedge by bidding on several items simultaneously may result in a bidder winning multiple items for which he has no use.

\footnotetext{
${ }^{1}$ An ascending proxy auction is an auction in which bidders submit a reservation price to a proxy agent that then bids on their behalf as if it were participating in an English auction-increasing the standing price by the minimum amount necessary to win the auction (provided that this does not exceed the entered reservation value). Such auctions are said to be 'hard-close' when there exists a fixed deadline after which no new or revised reservation bids are accepted.

${ }^{2}$ See, for example, Ariely, Ockenfels, and Roth (2005), Bajari and Hortaçsu (2003), Ely and Hossain (2009), and Roth and Ockenfels (2002) for empirical and experimental demonstrations of this phenomenon.

${ }^{3}$ See Bajari and Hortaçsu (2003) and Ely and Hossain (2009).

${ }^{4}$ With independent values, using proxy bids in this way ostensibly converts the procedure into a second price auction. This notwithstanding, there are equilibria of the single-unit proxy auction that involve sniping.
} 
The main contribution of this paper is to show that there exists a strategy-which I call defensive sniping - that both allows bidders to coordinate in price discovery and deters sniping. This strategy builds upon a well-known myopic bidding rule provided by Demange, Gale, and Sotomayor (1986). In particular I show that, whilst the myopic strategy is vulnerable to sniping, a modification in which all bidders submit an additional, large bid in the closing stages of the auction is not. Besides using an intuitive and straightforward bidding rule, the resulting equilibrium has the desirable properties of being efficient and belief-free. I show that any equilibrium with these attractive properties is equivalent to the defensive sniping strategy and, in particular, must have some kind of defensive element.

The results presented here suggest that the observed inefficiency in environments such as eBay might be remedied with better bidder education. Currently, eBay advises buyers to use the proxy system as follows: ${ }^{5}$

“... we suggest that you bid the maximum amount that you're willing to pay for an item..."

Left unqualified, this is problematic to the extent that it renders price discovery and coordination around the efficient outcome almost impossible. Indeed, there is a sizable body of evidence that suggests bidders are eschewing eBay's advice (and the proxy system) altogether, instead bidding 'naively' in an incremental fashion. ${ }^{6}$ I show that this behaviour creates opportunities for profitable sniping. However, the message that bidders should augment this naive strategy with defensive bidding is relatively straightforward and should be easy to communicate-even to relatively unsophisticated auction participants.

This paper is most closely related to work on bidding in simultaneous, heterogeneous item auctions. In addition to Demange, Gale, and Sotomayor (1986), who provide an algorithmic bidding rule that is a starting point for the analysis, results from the broader assignment literature are also of direct relevance. In particular, Leonard (1983) and Shapley and Shubik (1971) provide useful results on the properties of core allocations in such markets. Gul and Stacchetti (2000) establish that the algorithm of Demange, Gale, and Sotomayor (1986) forms a perfect Bayesian equilibrium of a simultaneous ascending auction with unit demand bidders when there is no fixed ending time.

Bajari and Hortaçsu (2004) provide a survey of a number of theoretical and empirical results on various issues connected to online auctions, many of which use a hard-close rule. More closely related to the present paper, recent work has recognised the significance of concurrency in Internet auctions. Peters and Severinov (2006) provide a theoretical model of online auction markets that have concurrent auctions for a homogeneous good. The authors model agents as arriving sequentially and incrementally bidding in

\footnotetext{
${ }^{5}$ See http://pages.ebay.co.uk/help/buy/bidding-overview.html (accessed 4th August 2011).

${ }^{6}$ See, for example, Roth and Ockenfels (2002) for survey evidence and Ely and Hossain (2009), Zeithammer and Adams (2010) for empirical results.
} 
a uniform-price fashion until they are declared the high bidder for one of the available items. In a primarily experimental paper, Ely and Hossain (2009) model bidding in a similar fashion. Unlike Ely and Hossain (2009) and Peters and Severinov (2006), I allow the various items for sale to be heterogeneous, which significantly complicates the allocation and equilibrium determination problem. ${ }^{7}$

A number of empirical studies speak to the importance of the dynamic and simultaneous structure of online proxy auctions. Zeithammer and Adams (2010) provide an empirical analysis of bidding patterns in eBay proxy auctions. They reject the sealed bid model of bidding in such auctions (which holds that these auctions can be modeled in the abstract as a second price sealed-bid auction), and show that the data is more consistent with a mixed model that has both sealed bidding and incremental bidding. In order to resolve the coordination problem in my model, bidders submit small bids across a number of items in a process of price discovery. This process also calls upon bidders to submit more than one bid per-item. Empirical evidence from Anwar, McMillan, and Zheng (2006) supports the prediction that agents in simultaneous auction environments bid across concurrent competing auctions in such a fashion, whilst Chiang and Kung (2005) and Wilcox (2000) find evidence for multiple-bidding in proxy auctions.

\section{MODEL}

Let $\mathbb{\square}=\{1,2, \ldots, I\}$ be bidders for items $\mathbb{K}=\{1,2, \ldots, K\}$. The items are sold by simultaneous proxy auction during an interval of time denoted $t \in[0, T]$, where $T$ is a fixed ending time. More precisely, denote by $\mathbf{b}_{i}(t)=\left(b_{i}^{1}(t), \ldots, b_{i}^{K}(t)\right)$ the vector of $i$ 's bids at time $t$. At each $t$, players chose some $\mathbf{b}_{i}(t) \in \mathbb{R}_{+}^{K}$ satisfying $\mathbf{b}_{i}(t) \geq \mathbf{b}_{i}\left(t^{\prime}\right) \forall t^{\prime}<t$. ${ }^{8}$ Player $i$ is named provisional winner for $k$ at time $t$ if $b_{i}^{k}(t)>\max _{j \neq i}\left(b_{j}^{k}(t)\right)$. Ties are broken instantaneously. Denote $k$ 's (unique) provisional winner at time $t$ by $w^{k}(t)$. Let $\mathbf{x}_{i}(t)=$ $\left(x_{i}^{1}(t), \ldots, x_{i}^{K}(t)\right)$ be the vector whose $k^{\text {th }}$ element is equal to 1 if $w^{k}(t)=i$, and 0 otherwise. A (provisional) allocation, $\mathbf{X}(t)$, is the matrix formed by concatenating the $\mathbf{x}_{i}(t)$ together.

Players observe the game's history, $\mathscr{H}(t)$, comprised of the provisional winner and the second highest bid for each $k$ at each $t .^{9}$ The second highest bid serves as a provisional price, which I denote by $\bar{b}^{k}(t)$-that being the $k^{\text {th }}$ element of the vector $\overline{\mathbf{b}}(t)$. Provisional winners' bids are not directly observed. Ultimately, each $k \in \mathbb{K}$ is allocated to $w^{k}(T)$. Prices are determined according to $\mathbf{p}=\overline{\mathbf{b}}(T)$. Any $k$ with $b_{i}^{k}(T)<r^{k} \forall i$ goes unallocated (has $\left.w^{k}(T)=\varnothing\right){ }^{10}$

\footnotetext{
${ }^{7}$ Even where items are homogeneous, de facto heterogeneity may be introduced by differences in seller reputation or the terms of sale.

${ }^{8}$ I use $\geq$ and $\leq$ as element-wise relations for vectors.

${ }^{9}$ The second highest bid is to be understood as meaning the highest reservation bid submitted by someone other than the provisional winner.

${ }^{10} \mathrm{~A}$ (hidden) reserve price, $r^{k}$, can be easily incorporated into the model by viewing the seller $s \notin \square$ as a bidder who bids $b_{s}^{k}(0)=r^{k}$. A posted reserve price can be modeled by viewing the seller as two such bidders,
} 
Every $i \in \llbracket$ has a vector of (private) valuations, $\mathbf{v}_{i}=\left(v_{i}^{1}, \ldots, v_{i}^{K}\right)$. Each $v_{i}^{k}$ is drawn from some distribution $F_{i}^{k}$ on a non-degenerate, bounded support, $\varphi^{k} \subset \mathbb{R}_{+}$. Values are allowed to be correlated across bidders, across items, or both. ${ }^{11}$ For the most part, and unless stated otherwise, the correlation structure of values is allowed to take an arbitrary form, but on occasion it will prove useful to invoke one of the following assumptions.

Assumption 1 (i) $v_{i}^{k}>\inf \varphi^{k} \Rightarrow v_{i}^{k}>\inf \left[\operatorname{supp} F_{j}^{k}\left(v_{j}^{k} \mid \mathbf{v}_{i}\right)\right] \forall j \neq i ;$ and (ii) $v_{i}^{k}<\sup \varphi^{k} \Rightarrow$ $v_{i}^{k}<\sup \left[\operatorname{supp} F_{j}^{k}\left(v_{j}^{k} \mid \mathbf{v}_{i}\right)\right] \forall j \neq i$.

Assumption $2 \operatorname{supp} F_{j}^{k}\left(v_{j}^{k} \mid \mathbf{v}_{i}\right)=\operatorname{supp} F_{j}^{k}\left(v_{j}^{k} \mid v_{j}^{m}\right)=\varphi^{k} \forall j \neq i \forall m \neq k$.

In words, both of these assumptions state that bidders' values are imperfectly correlated $^{12}$ in the sense that (conditional on observation of his own $\mathbf{v}_{i}$ ) a bidder, $i$, cannot typically be certain that his value for $k$ is above or below that of a given rival bidder. Note that Assumption 2 implies Assumption 1 and therefore represents a stronger restriction on the correlation structure-requiring that whilst $\mathbf{v}_{i}$ and $v_{j}^{m}$ may be informative about the relative likelihood of different $v_{j}^{k}$, they contain no new information about the range of values $v_{j}^{k}$ can take.

A strategy maps $\mathscr{H}(t)$ and $\mathbf{v}_{i}$ into bids $\mathbf{b}_{i}(t)$ for each $t \in[0, T]$. Utility is quasi-linear in money, and players are not budget constrained. Each bidder is assumed to have unit demand so that final utility is $U_{i}=\max _{k}\left(x_{i}^{k}(T) v_{i}^{k}\right)-\mathbf{p} \cdot \mathbf{x}_{i}(T) \equiv u_{i}-\mathbf{p} \cdot \mathbf{x}_{i}(T) .{ }^{13}$ Unit demand is a natural fit under a number of circumstances such as when bidders are consumers bidding for durable goods, or when the bidders are firms bidding for indivisible inputs (such as operating licenses) and the regulator has imposed that no firm may buy-up more than one input in order to stimulate after-market competition.

\section{BIDDING}

Define by $\gamma_{i}^{k}(t)$ the indicator function having value 1 for precisely one randomly chosen element of the surplus-maximising set of items:

$$
\mathbb{S}_{i}(t)=\left\{k: v_{i}^{k}>\bar{b}^{k}(t) \text { and } k \in \arg \max \left(v_{i}^{m}-\bar{b}^{m}(t)\right)\right\},
$$

and value 0 otherwise. If $\mathbb{S}_{i}(t)=\varnothing$ then $\gamma_{i}^{k}(t)=0 \forall k$. Thus, $\gamma_{i}(t)$ selects one of the "most appealing" items to $i$ at prices $\overline{\mathbf{b}}(t)$, where most appealing means having the largest difference between the bidder's value and the current second highest bid.

resulting in $\bar{b}^{k}(0)=r^{k}$. Provided that the reserve price is set in a manner consistent with efficiency, the result below transfer straightforwardly to such a set-up.

${ }^{11}$ This paper is primarily concerned with the existence of an equilibrium that is belief-free and is therefore quite robust to variations in the precise distributional properties of bidders' values.

${ }^{12}$ Note, in particular, that Assumptions 1 and 2 encompass independent values as a special case.

${ }^{13}$ Simultaneous ascending auctions cannot implement efficiency for general substitute preferences (Gul and Stacchetti, 2000). 
Now consider the myopic bid updating strategy given by $b_{i}^{k}(0)=0$, and the following rule:

$$
b_{i}^{k}(t+d t)=\left\{\begin{array}{l}
\left(\bar{b}^{k}(t)+\alpha_{i}^{k}(t) d t\right) \text { if } \gamma_{i}^{k}(t) \prod_{k \in \mathbb{K}}\left(1-x_{i}^{k}(t)\right)=1 \\
b_{i}^{k}(t) \text { otherwise }
\end{array}\right.
$$

with $\alpha_{i}^{k}(t)>0$ a function that controls the rate of bid updating for each $i, k, t$. I let $d t \rightarrow 0$, and assume that the $\alpha_{i}^{k}(t)$ are large enough to ensure that (1) achieves terminal prices before $T$. In words, (1) has $i$ increase his bid on some $k$ provided (i) he is not currently a provisional winner, and (ii) at the current bid level he extracts a positive surplus from $k$ that is at least as great as that from any other item.

At this juncture it is helpful to spend some time considering the form of a Walrasian (or competitive) equilibrium within the present framework. I use the following definition:

Definition 1 (Walrasian Equilibrium) An allocation $\mathbf{X}=\mathbf{x}_{1}, \ldots, \mathbf{x}_{I}$, and a price vector $\mathbf{p}$ constitute a Walrasian equilibrium if (i) $\sum_{i \in \square} x_{i}^{k} \leq 1 \forall k$, (ii) $p^{k}=0$ whenever $\sum_{i \in \square} x_{i}^{k}=0$, and (iii) $\max _{k}\left(x_{i}^{k}(T) v_{i}^{k}\right)-\left(\mathbf{x}_{i} \cdot \mathbf{p}\right) \geq \max _{k}\left(y_{i}^{k}(T) v_{i}^{k}\right)-\left(\mathbf{y}_{i} \cdot \mathbf{p}\right)$ for all $i$, and any $\mathbf{y}_{i}$ with $y_{i}^{k} \in$ $\{0,1\} \forall k$.

These conditions respectively require that no item is allocated more than once, that unallocated items have a price of zero, and that-taking the Walrasian price as given-no buyer can do better than his Walrasian allocation. ${ }^{14}$ Sellers play no part in this definition and, consequently, condition (ii) is required in order to rule out the sort of unattractive allocations in which all prices are prohibitive and no player has (or wants to have) any item. With this definition in mind, it becomes possible to introduce lemma 1 as follows:

Lemma 1 In the $d t \rightarrow 0$ limit, the algorithm proposed in (1) terminates in a Walrasian allocation with corresponding supporting price vector.

\section{Proof. See appendix.}

There will generally be a multitude of price vectors that are capable of supporting a Walrasian equilibrium. For example, if $I=K=2$ and $\mathbf{v}_{1}=(2,1), \mathbf{v}_{2}=(1,2)$ then any $\mathbf{p}$ such that $p^{1}, p^{2} \in[0,2]$ and $\left|p^{1}-p^{2}\right| \leq 1$ supports the Walrasian equilibrium allocation with $w^{1}=1, w^{2}=2$. Given this multiplicity, one might ask: is there any logic to the means by which (1) chooses an equilibrium price vector? The answer is affirmative: the (Walrasian) allocation reached by (1) is supported by a price vector which is small in the strong sense that each item price, $p^{k}$, is no greater than the corresponding price from

\footnotetext{
${ }^{14}$ Note that, when bidders have unit demand, condition (iii) requires that no bidder is allocated more than one item with $p^{k}>0$. Condition (iii) also demands that bidders prefer their Walrasian allocation and price to the outside option of reneging on the agreement to buy and receiving the null allocation.
} 
any other Walrasian price vector. ${ }^{15}$ I denote this minimum Walrasian price vector by $\underline{\mathbf{p}}$ where, in particular, if there exists a $k$ such that $p^{k}<\underline{p}^{k}$ then $\mathbf{p}$ must produce excess demand for some $k$.

Lemma 2 The competitive equilibrium reached by (1) is supported by $\underline{\mathbf{p}}$, the smallest price vector capable of supporting a Walrasian equilibrium in the market.

Proof. See appendix.

A version of this convergence result was first demonstrated by Demange, Gale, and Sotomayor (1986) in the context of standard (open-ended) English auctions. This result is of special interest since $\underline{\mathbf{p}}$, and the corresponding Walrasian allocation have been shown by Leonard (1983) to be identical to the Vickrey Clarke Groves (VCG) outcome. Demange, Gale, and Sotomayor (1986) did not consider the incentive properties of their bidding rule, but Gul and Stacchetti (2000) established that symmetric myopic bidding in a similar fashion to (1) is a perfect Bayesian equilibrium (PBE) of a simultaneous English auction whenever $\underline{\mathbf{p}}$ and the VCG price vector coincide in this fashion.

The main implication of instituting a fixed end time, $T$, is that agents may place a final bid (at time $T$ ) to which no rival has time to respond. In Lemma 4, I establish that this undermines the ability of myopic bidding to support an equilibrium in such an environment. I begin, though, with the following result:

Lemma 3 If $\underline{\mathbf{p}}_{-i}$ is the minimum Walrasian price in a market with buyers $\llbracket \backslash i$ then $\underline{\mathbf{p}} \geq$ $\underline{\mathbf{p}}-i$

Proof. If $\left\{k: \underline{p}^{k}<\underline{p}_{-i}^{k}\right\} \neq \varnothing$ then, with set of players $\llbracket \backslash i, \underline{\mathbf{p}}$ must produce excess demand since $\underline{\mathbf{p}}_{-i}$ is a minimum Walrasian price. Since $i$ 's demand is non-negative and depends only on $i$ 's own valuations, $\underline{\mathbf{p}}$ also produces excess demand for $\mathbb{1}$. But then $\underline{\mathbf{p}}$ is not an equilibrium price vector.

Lemma 4 Under Assumption 1, the strategy defined in (1) does not constitute an equilibrium of the hard-close proxy auction.

Proof. If all players use (1) then $U_{i}=\max \left\{0, \max _{k}\left\{v_{i}^{k}-\underline{p}^{k}\right\}\right\}$. If, instead, $i$ abstains from bid updating during $[0, T)$ then (1) converges to $\underline{\mathbf{p}}_{-i}$. Thus, $i$ can win any $k$ at price $\underline{p}_{-i}^{k}$ with a single snipe bid at time $T$ to which rivals cannot respond. Lemma 3 implies $\mathrm{p} 54$ that abstention followed by a snipe bid is never worse than (1). To establish that (1) is not an equilibrium, it therefore suffices that there is some type, $\mathbf{v}_{i}$, of bidder that sometimes strictly gains by abstaining and then sniping. The existence of such a bidder type can be

\footnotetext{
${ }^{15}$ Shapley and Shubik (1971) provide an existence proof for such a 'smallest Walrasian price vector' in the unit demand setting.
} 
demonstrated by construction of a generic example. Fix values according to the following conditions:

$$
v_{l}^{k}<v_{j}^{k} \leq v_{i}^{k} \forall l \neq j, i ; \quad v_{l}^{m}>v_{j}^{m} \forall m \neq k \forall l \neq j,
$$

where $k \in \arg \max _{m \in \mathbb{K}}\left\{v_{i}^{m}-\underline{p}^{m}\right\}$ ( $k$ is $i$ 's efficient item). It is clear that $\underline{p}^{k}=v_{j}^{k}$ and (1) thus induces $U_{i}=v_{i}^{k}-v_{j}^{k}$, whilst $\underline{p}_{-i}^{k} \leq \max _{l \neq i, j} v_{l}^{k}<v_{j}^{k}$ so that abstention followed by a snipe bid is a strictly profitable deviation for $i$ when (2) is satisfied. Assumption 1 implies that, given $v_{i}^{k}>\inf \varphi^{k}$ for some $k$, (2) is satisfied with positive probability, and every $i$ with $v_{i}^{k}>\inf \varphi^{k}$ for some $k$ obtains a strictly higher expected payoff from sniping. ${ }^{16}$

Players that use a myopic bidding rule in a hard-close auction are typically described as naive since they behave as one might expect them to do in a standard English auction, without acknowledging the strategic considerations introduced by the fixed ending time. Such naive behaviour is open to exploitation, as demonstrated in Lemma 4; indeed, Ockenfels and Roth (2006) and Ely and Hossain (2009) have shown in a less general setting that sniping (last-minute bidding) is a best response to such behaviour. It is not immediately obvious that this should be true in the heterogeneous good environment. In particular, bidders using (1) adjust their bidding pattern in response to shifts in the relative prices of the items in a manner that depends upon their $\mathbf{v}_{i}$, and it is not clear whether $i$ can construct some sequence of bids that manipulates the algorithmic substitution across items and the evolution of prices so that he is better off than when sniping. Proposition 5 establishes that he cannot. Specifically, let pure sniping be the strategy that has a bidder update his bid only at time $T$, bidding $b_{i}^{k}(T)=v_{i}^{k}$ on one $k \in \mathbb{S}_{i}(T-d t)$. The following result obtains:

Proposition 5 Pure sniping is a best response to the naive myopic bidding given in (1).

Proof. When using a pure sniping strategy against rivals who use (1), $i$ is able to buy any $k \in \mathbb{K}$ at a price of $\underline{p}_{-i}^{k}$ so that his payoff is $\max \left\{0, \max _{k}\left(v_{i}^{k}-\underline{p}_{-i}^{k}\right)\right\}$. Suppose that $i$ uses some other strategy. Clearly, if $i$ abstains from updating his bid during $[0, T)$, he can do no better than $\max \left\{0, \max _{k}\left(v_{i}^{k}-\underline{p}_{-i}^{k}\right)\right\}$, regardless of his choice of time $T$ bid. Suppose, instead, that $i$ uses some strategy that has him bid during [0,T) (possibly in addition to bidding at time $T$ ), whilst his rival bidders continue to use (1). Denote by $\widehat{\mathbf{X}}$ the allocation that results when $i$ uses his deviation strategy during $[0, T)$ but abstains from bid updating at time $T$, and by $\widehat{\mathbf{p}}$ the associated price vector. Thus $i$ extracts a surplus that is, at most, equal to $\max \left\{0, \max _{k \in \mathbb{K}}\left(v_{i}^{k}-\widehat{p}^{k}\right)\right\} .{ }^{17}$

\footnotetext{
${ }^{16}$ Note that if the $v_{i}^{k}$ are atomlessly distributed on intervals of $\mathbb{R}_{+}$then $v_{i}^{k}>\inf \varphi^{k} \forall m \neq k$ is satisfied with probability 1 and every bidder finds that sniping yields higher expected utility than does compliance with the putative myopic bidding equilibrium.

${ }^{17}$ The actual surplus may be somewhat less than this if, for example, $i$ uses a strategy that calls upon him to make a large and early bid-leaving him committed to winning an item that is ultimately suboptimal.
} 
Now, consider the valuation vectors given by

$$
\begin{aligned}
& v_{\omega(k)}^{k}=\left\{\begin{array}{l}
0 \text { if } \hat{x}_{i}^{k} \neq 1 \\
\sup \varphi^{k}+1 \text { if } \widehat{x}_{i}^{k}=1,
\end{array}\right. \\
& v_{I+k}^{m}=\left\{\begin{array}{l}
\min \left\{b_{i}^{m}(T), \bar{b}^{m}(T)\right\} \text { if } m=k \\
0 \text { if } m \neq k,
\end{array}\right.
\end{aligned}
$$

with $\{\omega(1), \ldots, \omega(K), I+1, \ldots, I+K\} \cap \mathbb{q}=\varnothing$. If bidders $\{\omega(1), \ldots, \omega(K), I+1, \ldots, I+K\}$, having such utility functions, were to bid in $i$ 's place, and do so according to (1) then the outcome obtained at time $T$ would be similar to that produced by the arbitrary strategy used by $i$ during $[0, T)$; having the same price vector, $\widehat{\mathbf{p}}$, with $i$ 's (provisionally allocated) items assigned instead to the $\omega(k)$ s. Since all members of $\{\omega(1), \ldots, \omega(K), I+1, \ldots, I+K\} \cup \square \backslash i$ are using (1), $\widehat{\mathbf{p}}$ must be the minimum Walrasian equilibrium price for the market with this set of bidders. It must, then, be true that $\widehat{p}^{k} \geq \underline{p}_{-i}^{k}$ because adding players can never lower the minimum Walrasian price (Lemma 3 ).

An important caveat attached to the behaviour described in Proposition 5 is that the surplus maximising item for the sniper need not be the same item that he would otherwise have been allocated by the mechanism viz. his socially efficient allocation. Example 1 demonstrates how the incentive to snipe can induce this kind of inefficiency.

Example 1 Consider valuation vectors given as follows

$$
\mathbf{v}_{1}=(10,10) ; \mathbf{v}_{2}=(5,0) ; \mathbf{v}_{3}=(5,0) ; \mathbf{v}_{4}=(0,15)
$$

The efficient assignment is $w^{1}(T)=1$ and $w^{2}(T)=4$ with a total social surplus of 25 (minimum Walrasian prices are $p^{1}=5, p^{2}=5$ ). However, if 1 uses the pure sniping strategy against rivals using (1) then he is faced with $\overline{\mathbf{b}}(T-d t)=(5,0)$ and prefers to win item 2 at a price of zero yielding $w^{1}(T) \in\{2,3\}, w^{2}(T)=1$, and a total social surplus of 15 .

Thus, bidding of the form described by (1) is vulnerable to opportunistic sniping behaviour. The behaviour described in Proposition 5 and illustrated in Example 1 does not, however, constitute an equilibrium of the hard-close auction game when all players are fully-rational. In particular, as example 1 shows, those players that bid algorithmically face a positive probability of being outbid by the sniper, even when their valuation for their respective efficient items is higher than that of all of their rivals. The risk of surplus loss due to usurpation can be partially mitigated if the algorithmic bidders also submit a late bid equal to their value on the item to which they are allocated by the algorithm. I call this kind of bidding 'defensive' and refer to the algorithmic strategy augmented with a defensive element as 'defensive sniping': ${ }^{18}$

\footnotetext{
${ }^{18}$ It should be emphasised that the 'late' bid submitted as part of a defensive sniping strategy may be
} 
Definition 2 Defensive sniping is a strategy that has $i$ bid using (1) during [0,T), and then according to

$$
b_{i}^{k}(T)=\left\{\begin{array}{l}
v_{i}^{k} \text { if } w^{k}(T-d t)=i \\
b_{i}^{k}(T-d t) \text { otherwise }
\end{array}\right.
$$

at time $T$.

Returning to Example 1, if player 4 uses the defensive sniping strategy and bids $b_{4}^{2}(T)=15$ then, in the face of pure sniping by player 1 , he extracts a surplus of 5 from item 4-still worse than the efficient allocation at $\underline{\mathbf{p}}$ that leaves him with a surplus of 10 , but non-the-less better than the outcome if he bids myopically and receives zero surplus.

The adoption of the defensive sniping strategy by players $j \in \llbracket \backslash i$ undermines the advantage of the pure sniping strategy for $i$ since the latter strategy exploits the general differential between the highest algorithmic bid placed by the provisional winner for each item and the valuation of that same bidder. The snipe portion of the defensive sniping strategy has the effect of eliminating this differential. In example 1, sniping against naive rivals produces a positive increase in surplus of 5 for player 1 , but using the same strategy when all rivals switch to defensive sniping results in a fall in surplus of 5 for player 1 vis-à-vis the efficient outcome.

More generally, once players adopt the defensive sniping strategy, $i$ must take as given the fact that he will face a valuation bid on every $k$ for which $w^{k}(T-d t) \in \mathbb{}$. Since other players' valuations are invariant to observed bidding behaviour, $i$ 's primary strategic concern is to ensure that he is allocated his surplus maximising item before $T$. There is thus hope that an efficient equilibrium with symmetric, algorithmic strategies might exist and indeed the existence of such an equilibrium is confirmed in Proposition 6. The proof of Proposition 6 establishes that any deviation from defensive sniping is not only non-profitable in expectation, but also non-profitable for any realised profile of rival types. Aside from being efficient, the equilibrium characterised in Proposition 6 is therefore belief-free in the following sense:

Definition 3 An equilibrium is belief-free if, starting from an arbitrary price vector, $\overline{\mathbf{b}}(t)$, each $i$ has a continuation strategy that is optimal against every $\left\{\mathbf{v}_{j} \in \times_{k} \varphi^{k}\right\}_{j \in \mathbb{} \text { iviven the }}$ other bidders' continuation strategies.

Thus, in a belief-free equilibrium, each bidder would never wish to change his strategy, even given complete knowledge of the other bidders' types (provided that those bidders continue to play according to the equilibrium).

submitted at any $t$ after the algorithm has converged on terminal prices. For concreteness, I take the time of this bid to be $T$. Introducing stochastic bidder arrival can encourage bidders to delay their defensive snipe bid by creating the possibility that a new bidder arrival will cause algorithmic bidding to restart (and the relative item prices to change). 
Proposition 6 Symmetric use of the defensive sniping strategy constitutes a belief-free equilibrium of the hard-close auction game.

The proof of Proposition 6 is divided across two sub-cases detailed in the following two lemmas. Firstly, any deviation by $i$ that results in $\sum_{\mathbb{}} x_{i}^{k}=0$ is clearly weakly worse than compliance with the defensive sniping equilibrium. Now, for concreteness, consider a deviation by $i$ in which $w^{1}(T)=i$. Moreover, suppose that $\operatorname{argmax}_{k}\left\{v_{i}^{k}: w^{k}(T)=i\right\}=1$ so that when $i$ wins multiple items the unit he consumes is that labeled 1 . This is without loss of generality. Use a tilde to denote deviation variables, and a hat to denote the outcome that would prevail if $i$ followed his deviation strategy during $[0, T)$, but abstained from bid updating at time $T$.

Lemma 7 Suppose that players $j \in \mathbb{Q} \backslash i$ use a defensive sniping strategy and consider a deviation by $i$ that results in $\widetilde{w}^{1}(T)=i$. If $\widehat{w}^{1}(T)=i$ or if $\widetilde{p}^{1}=0$ then $i$ is no better off than if he had also used a defensive sniping strategy.

Proof. If $\widehat{w}^{1}(T)=i$, or if $\widetilde{p}^{1}=0$, then, $\widehat{p}^{1}=\widetilde{p}^{1}$ and $v_{j}^{1}-\widehat{p}^{1} \leq \max \left\{0, \max _{k \neq 1}\left\{v_{j}^{k}-\widehat{p}^{k}\right\}\right\} \forall j \neq$ $i$. Begin by considering the case with $\widetilde{\mathbf{x}}_{i}(T)=(1,0,0, \ldots, 0)$. The deviation payoff for $i$ is then $v_{i}^{1}-\widetilde{p}^{1}$. Consider an artificial player, $\psi$, having valuations:

$$
v_{\psi}^{k}=\left\{\begin{array}{l}
0 \text { if } k \neq 1 \\
\sup \varphi^{k}+1 \text { if } k=1
\end{array}\right.
$$

Note that $\widehat{\mathbf{p}}$ is a Walrasian price in the market with $\psi \cup \square \backslash i$. Moreover, when $i$ imitates $\psi$ in myopic bidding against $\square \backslash i$, he wins item 1 at its minimum Walrasian price in this market, $\underline{\mathbf{p}}_{\psi} \leq \widehat{\mathbf{p}}$. Since the VCG mechanism is incentive compatible, it follows that $\max \left\{0, \max _{k}\left\{v_{i}^{k}-\underline{p}^{k}\right\}\right\} \geq v_{i}^{1}-\underline{p}_{\psi}^{1} \geq v_{i}^{1}-\widehat{p}^{1}=v_{i}^{1}-\widetilde{p}^{1}$.

If $\sum_{\mathbb{K}} \widetilde{x}_{i}^{k}>1$ and $\widehat{w}^{1}(T)=i$ or $\widetilde{p}^{1}=0$ then the deviation payoff for $i$ is $v_{i}^{1}-\widetilde{p}^{1}-\sum_{\mathbb{K} \backslash 1} \widetilde{x}_{i}^{k} \widetilde{p}^{k}$, so it suffices to show that $v_{i}^{1}-\widetilde{p}^{1}=v_{i}^{1}-\widehat{p}^{1} \leq \max \left\{0, \max _{k \in \mathbb{K}}\left(v_{i}^{k}-\underline{p}^{k}\right)\right\}$. Introduce a collection of new bidders with valuations given by

$$
v_{I+k}^{m}=\left\{\begin{array}{l}
\widehat{b}_{i}^{m}(T) \text { if } m=k \\
0 \text { if } m \neq k
\end{array}\right.
$$

and consider the market consisting of players $\psi \cup\{I+2, \ldots, I+K\} \cup \square \backslash i$. Note that $\widehat{\mathbf{p}}$ is a Walrasian price in this market. The proof is then completed using the same logic as above and noting that imitating $\psi$ in symmetric defensive sniping against rival bidders $\{I+2, \ldots, I+K\} \cup \square \backslash i$ yields weakly lower payoffs than does doing so against $\square \backslash i$ (by Lemma $3)$.

Lemma 8 Suppose that players $j \in \mathbb{Q} \backslash i$ use a defensive sniping strategy and consider a deviation by $i$ that results in $\widetilde{w}^{1}(T)=i$. If $\widehat{w}^{1}(T) \neq i$ and $\widetilde{p}^{1}>0$ then $i$ is no better off than if he had used a defensive sniping strategy. 
Proof. The deviation payoff for $i$ is $v_{i}^{1}-\widetilde{p}^{1}-\sum_{\mathbb{K} \backslash 1} x_{i}^{k} \widetilde{p}^{k}$. If $\widehat{w}^{1}(T) \neq i$ and $\widetilde{p}^{1}>0$ then it must be that $\widetilde{p}^{1}=v_{j}^{1}, j \neq i$. Now consider the market consisting of players $\psi \cup\{I+$ $2, \ldots, I+K\} \cup \square \backslash i$ (where $\psi$ and $I+k$ have values given in (3) and (4) respectively), who all bid according to (1). Player $\psi$ wins item 1 at the VCG price in this market so that $\psi$ 's payment is

$$
\underbrace{\max _{\text {allocating } \mathbb{K}} \sum_{l \neq \psi} u_{l}}_{A}-\underbrace{\underset{\text { allocating } \mathbb{K} \backslash 1}{\max } \sum u_{l \neq \psi}}_{B}=v_{j}^{1}+\underbrace{\max _{\text {allocating } \mathbb{K} \backslash 1} \sum_{l \neq \psi, j} u_{l}}_{C}-\underbrace{\max _{\text {allocating } \mathbb{K} \backslash 1} \sum_{l \neq \psi} u_{l}}_{B} \leq v_{j}^{1},
$$

where the equality follows from the fact that $j$ is assigned item 1 by (1) under set of players $\{I+2, \ldots, I+K\} \cup \llbracket \backslash i$. The inequality follows from the fact that one possibility for calculating $B$ has $\sum_{k} x_{j}^{k}=0$, and all other player's allocations unchanged from those in $A$-whence $B=C$. It may, however, be better to allocate to $j$ some $k \in \mathbb{K} \backslash 1$, so that $C \leq B$. Now we have

$$
\begin{aligned}
\max \left\{0, \max _{k \in \mathbb{K}}\left(v_{i}^{k}-\underline{p}^{k}\right)\right\} \geq & i \text { 's surplus when imitating } \psi \text { in VCG against } \llbracket \backslash i \\
\geq & i \text { 's surplus when imitating } \psi \text { in VCG against players } \\
& \{I+2, \ldots, I+k\} \cup \llbracket \backslash i \\
\geq & v_{i}^{1}-v_{j}^{1}-\sum_{\mathbb{K} \backslash 1} x_{i}^{k} \widetilde{p}^{k} \\
= & i \text { 's deviation surplus, }
\end{aligned}
$$

where the first inequality follows from the VCG mechanism's incentive compatibility, the second from Lemma 3, and the third from the argument above.

Thus, regardless of the configuration of rival values, any deviation for $i$ is outperformed by the defensive sniping strategy when those rivals also defensively snipe. The mechanics of the proof are as follows: any deviation by a bidder that causes the auction's outcome to change results in a surplus for that bidder that can be bounded above by the surplus obtained by imitating an artificial bidder who bids according to (1) against an enlarged set of rivals. The incentive compatibility of the VCG mechanism, along with Lemma 3 then ensures that the deviation is non-profitable. Since $\underline{\mathbf{p}}$ and the corresponding allocation are the products of a VCG mechanism, efficiency of the defensive sniping equilibrium is immediate.

Corollary 9 The equilibrium described in Proposition 6 is efficient.

The belief-free nature of the defensive sniping equilibrium implies that it is not necessary to restrict belief updating to be consistent with $\mathscr{H}(t)$ in a Bayesian fashion (or, indeed, to place any requirements on beliefs at all). This not only makes the bidder's strategic problem extremely simple, but also makes the entire equilibrium more robust to mistakes by other players. Moreover, this equilibrium does not require that bidders know the distributions from which others' values are drawn. The low information re- 
quirements and intuitive bidding strategy make this equilibrium particularly attractive for practical implementation.

One might ask whether there are other, substantively different bidding behaviours that also support an efficient, belief-free equilibrium. Proposition 10, below, and the ensuing discussion establishes that, subject to some mild additional assumptions, every efficient, belief-free equilibrium is essentially equivalent to the defensive sniping equilibrium discussed above in the sense that it deterministically converges to the same VCG prices via myopic, incremental bidding. I say 'essentially equivalent' because there are a lot of slight variations on the defensive sniping strategy that are substantively equivalent to it. For example, if $K=2$ and $i$ 's values are $v_{i}^{1}>v_{i}^{2}$ then, at the start of the auction, algorithmic bidding as defined in (1) calls upon $i$ to repeatedly increment his bid on the first item so long as its price is below $v_{i}^{1}-v_{i}^{2}$. This is essentially equivalent to submitting a single proxy bid of $b_{i}^{1}(0)=v_{i}^{1}-v_{i}^{2}$, and allowing the proxy system to implement the incremental bidding on his behalf. I refer to such bids as pseudo-incremental since they correspond to a concise implementation of incremental bidding. These kinds of non-substantive variations notwithstanding, all efficient, belief-free equilibria fall into the template of the defensive sniping strategy.

To establish this result it will be useful to define $\bar{t} \equiv \inf \{t: \overline{\mathbf{b}}(t)=\overline{\mathbf{b}}(t+\Delta) \forall \Delta>0\}$, the time at which price discovery/algorithmic bidding terminates. Note that after $\bar{t}$ prices do not change and therefore neither to the $w^{k}$ s. It follows that, in an efficient equilibrium, the efficient allocation and a supporting price must have been reached by $\bar{t}$ and that $b_{i}^{k}(\bar{t}+\Delta)>\bar{b}^{k}(\bar{t}) \Rightarrow w^{k}(T)=i$. The following will also be useful:

Assumption 3 Each $\varphi^{k}$ is an interval of $\mathbb{R}_{+}$, and at least one of the following two conditions is satisfied for each $k$ : (i) $\inf \varphi^{k} \notin \varphi^{k}$, or (ii) $\bar{b}^{k}(0)=\inf \varphi^{k}$.

In words Assumption 3 says that it must be the case for each item either (i) that the support of the buyers' value distribution does not include its lower boundary, or (ii) that there is a reserve price equal to the minimum possible buyer valuation. Note that the second of these alternatives encompasses the standard case in which $\varphi^{k}=\left[0, \bar{v}^{k}\right]$ and there is no reserve price (i.e. $\bar{b}^{k}=0$ ). It is now possible to demonstrate that the defensive sniping equilibrium is essentially unique within the class of efficient, belieffree equilibria as follows:

Proposition 10 Under Assumptions 2 and 3, any efficient, belief-free equilibrium of a simultaneous ascending proxy auction must (a) have $\mathbf{p} \geq \mathbf{v}_{i}$ whenever $\sum_{k} x_{i}^{k}=0$; (b) be myopic in the sense that $w^{k}(t)=i$ only if $k \in \mathbb{S}_{i}(t)$; (c) be pseudo-incremental in the sense that, for all $t<\bar{t}$, each player's bids satisfy $v_{i}^{k}-v_{i}^{m} \geq b_{i}^{k}(t)-\bar{b}^{m}(t-d t) \forall k, m$; and (d) be algorithmic in the sense that it deterministically converges to price $\underline{\mathbf{p}}$. 
Proof. To prove part (a) suppose that the converse were true, which implies that $\exists k$ such that $b_{i}^{k}(T) \leq \widetilde{p}^{k}<v_{i}^{k}, \sum_{m \in \mathbb{K}} x_{i}^{m}=0$, where $\widetilde{p}^{k}$ is the resulting price for item $k$. Moreover, if $i$ 's strategy is belief-free, it must always be true that $b_{j}^{k}(T) \geq v_{i}^{k}>b_{h}^{k}(T) \forall h \neq j$ for one $j \neq i$-otherwise, $i$ would sometimes be better off with $b_{i}^{k}(T)=v_{i}^{k}$. Now suppose that we replace $j$ with bidder $\mathscr{J}$, where

$$
v_{\mathscr{J}}^{m}\left\{\begin{array}{l}
\in\left(\widetilde{p}^{k}, v_{i}^{k}\right) \text { if } m=k \\
<\min _{\Downarrow} v_{i}^{m} \text { if } m \neq k \text { and } \min _{\Downarrow} v_{i}^{m}>\inf \varphi^{m} \\
=\inf \varphi^{m} \text { if } m \neq k \text { and } \min _{\rrbracket} v_{i}^{m}=\inf \varphi^{m}
\end{array}\right.
$$

and consider the outcome when all $\mathscr{J} \cup \square \backslash j$ comply with the putative equilibrium. Since the equilibrium is efficient by hypothesis, $\mathscr{J}$ must either be allocated no item or be allocated item $m$ for which $v_{\mathscr{J}}^{m}=\inf \varphi^{m}=\bar{b}^{m}(0)$ (by Assumption 3), and therefore receives a payoff of zero. However, $\mathscr{J}$ 's strategy cannot then be belief-free since he can obtain a payoff of $v_{\mathscr{J}}^{k}-\widetilde{p}^{k}>0$ against bidders $\llbracket \backslash j$ by imitating $j$ 's strategy.

Part (b) is implied by part (c). To prove part (c) suppose that $v_{i}^{k}-b_{i}^{k}(t)<v_{i}^{m}-\bar{b}^{m}(t-d t)$ for some $m \in \mathbb{K} \backslash k, t<\bar{t}$. In a belief-free equilibrium, every continuation $\mathbf{b}_{i}(t)$ must be optimal against every possible $\left\{\mathbf{v}_{i}\right\}_{\llbracket \backslash i}$. Fix values so that $\mathbf{v}_{h} \leq \overline{\mathbf{b}}(t-d t) \forall h \neq i, j ; v_{j}^{l} \leq \bar{b}_{j}^{l}(t-$ $d t) \forall l \neq k$; and $v_{j}^{k}=\min \left\{\sup \varphi^{k}, b_{i}^{k}(t)\right\}-\epsilon$. Since the equilibrium price of each $l \neq k$ cannot then rise and the equilibrium price of $k$ cannot exceed $b_{i}^{k}(t), i$ 's strategy is only optimal if $\bar{b}^{k}$ is and remains below $v_{i}^{k}-v_{i}^{m}+\bar{b}^{m}(t-d t)$, which in turn places a ceiling on how high $b_{j}^{k}$ can get. However, $j$ 's strategy can then not be consistent with part (a) for $\epsilon \rightarrow 0$.

Now, consider the three parts of Definition 1. As in the proof of Lemma 1, parts (i) and (ii) of the definition are always satisfied in the ascending proxy auction. Given that, by part (b) of this proof, $w^{k}(t)=i \Rightarrow k \in \mathbb{S}_{i}(t)$, every winning bidder prefers his allocation to any alternative at price p. Moreover, by part (a) of this proof all unsuccessful bidders can do no better than accepting the null allocation at price $\mathbf{p}$. Thus, part (iii) of Definition 1 is also satisfied and an efficient, belief-free equilibrium must result in a Walrasian outcome. The proof of Lemma 2 can now be applied to establish part (d).

Proposition 10 establishes that any efficient, belief-free equilibrium bidding must be myopic in the sense that bidders only ever hold the provisional winner position for items in their myopically surplus-maximising set, and pseudo-incremental in the sense that the increments in bidders' bids are bounded above in magnitude by the amount of surplus those bidders are able to achieve from the next best alternative. This result applies to simultaneous proxy auctions with or without a fixed ending time.

Of course, once the price discovery phase is over and efficient items have been identified, defensive bidding need not be incremental. In particular, note that part (c) of Proposition 10 applies only for $t<\bar{t}$; after $\bar{t}$ bidders can use a coordination device such as 'if item prices have remained unchanged for a period of length $\Delta t$ then only bid de- 
fensively on items for which I am already the high bidder. ${ }^{, 19}$ If such a strategy is in use by other bidders then $i$ can bid up to $v_{i}$ on his efficient item in a belief-free fashion with impunity after $\bar{t}+\Delta t$ has elapsed.

Indeed, part (d) of proposition 10 also implies that any putative equilibrium bid profile that is belief-free, implements the efficient allocation, and has no defensive element (i.e. that has $\left.b_{w^{k}(T-d t)}^{k}(T)=\underline{p}^{k}\right)$ is vulnerable to sniping in the fashion made clear by Lemma 4 and thus cannot be an equilibrium. To yield an efficient, belief-free equilibrium, any such strategy profile must therefore be augmented with a defensive element (must have $b_{w^{k}(T-d t)}^{k}(T)>p^{k}$ whenever $\left.v_{w^{k}(T-d t)}^{k}>\underline{p}^{k}\right)$ when a hard-close rule is in effect. Proposition 6 showed that, for any deviation and any rival valuation profiles, a defensive bid of $b_{w^{k}(T-d t)}^{k}(T)=v_{w^{k}(T-d t)}^{k}$ can serve this purpose.

More generally, write $w^{k}$ for the efficient winner of item $k$ and $w_{-}^{k}$ for the bidder who would win $k$ if $w^{k}$ were not to participate. The surplus accruing to $w^{k}$ is $U_{w^{k}}=v_{w^{k}}^{k}-\underline{p^{k}}$, where

$$
\underline{p}^{k} \equiv v_{w_{-}^{k}}^{k}+\underbrace{\max _{\text {allocating } \mathbb{K} \backslash k} \sum_{l \neq w^{k}, w_{-}^{k}} u_{l}-\max _{\text {allocating } \mathbb{K} \backslash k} \sum_{l \neq w^{k}} u_{l}}_{\leq 0}
$$

is the minimum Walrasian (VCG) price for item $k .^{20}$ If, on the other hand, $w^{k}$ does not participate in the equilibrium, but rather uses the pure sniping strategy then he obtains a surplus of at least $v_{w^{k}}^{k}-b_{w_{-}^{k}}^{k}(T)$, where $b_{w_{-}^{k}}^{k}(T)$ is the defensive bid of $w_{-}^{k}$. For the putative equilibrium to be belief-free, it must be true that compliance with the equilibrium by $w^{k}$ is at least as profitable as the pure sniping strategy for every configuration of rival values, which requires, in particular, that $b_{w^{k}}^{k}(T) \geq \underline{p}^{k}$ always holds; $\underline{p}^{k}$ is therefore a lower bound on an effective defensive bid for $w_{-}^{k}$ in such an equilibrium, and a similar argument can be made for the other $k$. However, it will typically be impossible for $w_{-}^{k}$ to calculate $\underline{p}^{k}$ based only on observations of the $\overline{\mathbf{b}}$ and $\mathbf{X}$ that result when $w^{k}$ abstains. More to the point-in keeping with the spirit of the belief-free solution concept-bidders' defensive bids should not be based on calculations involving (beliefs about) other bidders' values. It is therefore interesting to ask: what is the lowest $b_{w_{-}^{k}}^{k}(T)$ that is guaranteed to always satisfy $b_{w_{-}^{k}}^{k}(T) \geq \underline{p}^{k}$, regardless of rivals' values? It is fairly clear from (5) that this 'minimum defensive bid' is equal to $v_{w_{-}^{k}}^{k}$. Thus, whilst a defensive bidding strategy of $b_{w_{-}^{k}}^{k}(T)=v_{w_{-}^{k}}^{k}$ is always sufficient to deter sniping, any lower defensive bid would sometimes leave open profitable opportunities for such opportunistic behaviour.

Remark 11 Under Assumptions 2 and 3, every efficient, belief-free equilibrium must in-

\footnotetext{
${ }^{19}$ An alternative coordination device, used in Definition 2 above, is to only bid defensively at $T$.

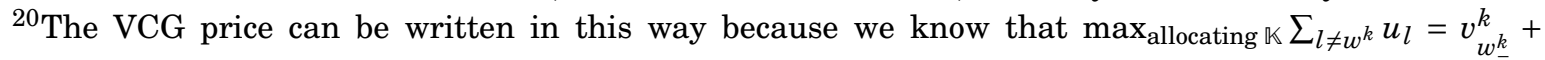
$\max _{\text {allocating } \mathbb{K} \backslash k} \sum_{l \neq w^{k}, w_{-}^{k}} u_{l}$.
} 
volve defensive bidding. The smallest defensive bid that is always sufficient to deter sniping is $b_{w^{k}(T-d t)}^{k}(T)=v_{w^{k}(T-d t)}^{k}$.

More generally, since any efficient equilibrium has to be payoff equivalent to the defensive sniping equilibrium (see Krishna and Perry, 2000; Williams, 1999), the expected price in such an equilibrium must be equal to $\underline{\mathbf{p}}$. Thus, if winning bidders increment the price of the item they win by just enough to ensure victory then the expected high bid will also be equal to $\mathbf{p}$, which creates gains in expectation from sniping (more precisely, from imitation of a type $\mathbf{v}=\left(\inf \varphi^{1}, \ldots, \inf \varphi^{K}\right)$ bidder followed by a last-minute snipe bid $)$. It follows that any efficient equilibrium that is not vulnerable to sniping must have some kind

of defensive element. As in the belief-free case, defensive bids of $b_{w^{k}(T-d t)}^{k}(T)=v_{w^{k}(T-d t)}^{k}$ always suffice to render this kind of deviation unprofitable in expectation.

\section{Discussion \& Conclusion}

There exists an efficient, belief-free equilibrium of simultaneous, hard-close proxy auctions when bidders have unit demand. Thus, fixed ending times need not always be regarded with suspicion. Indeed, if certainty regarding the end time is of value, if the seller fears that some bidders have an interest in unduly prolonging the auction, or if circumstances impose a natural deadline upon proceedings, then a hard-close rule may be a viable alternative to minimum bid increments or rules on the minimum rate of bid updating in ensuring a timely conclusion to bidding. The equilibrium is also robust to entry by new bidders, provided that bidders do not arrive too late. Being belief-free, equilibrium bids follow a simple rule that requires minimal knowledge and calculation, and can therefore be easily implemented. This is especially compelling because the leading practical implementation of ascending proxy auctions is in the consumer-to-consumer ecommerce space, where bidders are less likely to undertake a full-scale strategic analysis of the auction. In particular, it should be fairly straightforward for an auction operator such as eBay to communicate the efficient, belief-free strategy-and the need for defensive bidding in particular-to its participants.

It has been shown that when some bidders naively pursue a myopic strategy others will have an incentive to snipe, which is not generally efficient. This, again, serves to emphasise the importance of properly explaining the strategic nature of the auction to bidders. The environment considered in this article is also relatively friction-free. Where market frictions do exist they clearly have the potential to interfere with efficiency. For example, if some bidders arrive at the auction very close to its ending time then they may not have sufficient time to coordinate around the efficient allocation. Likewise, if bidding is costly then bidders may not be willing to submit enough bids to induce proper price discovery. In either case, knowledge that this may happen distorts the incentives of all other participants. 


\section{Appendix A OMitTed Proofs}

Proof of Lemma 1. Consider the three conditions in definition 1: (i) is always satisfied by the auction mechanism since $w^{k}(t)$ is defined as a singleton; to see that (ii) must be true, note that $p^{k}>0$ implies $b_{i}^{k}(T)>0$ for some subset of $\llbracket$ and, in turn, that $w^{k}(T) \in \llbracket$. Thus, by definition, $\sum_{i \in \square} x_{i}^{k} \neq 0$. Finally, for condition (iii): if $w^{k}(T)=i$ then, from (1), when $i$ last increased his bid on $k, \gamma_{i}^{k}$ was equal to 1 . Since the high bid for $k$ has not since risen, and the high bid for every $m \in \mathbb{K} \backslash k$ cannot have fallen, it must remain the case that

$$
\max _{m \in \mathbb{K}}\left(v_{i}^{m}-\bar{b}^{m}(T)\right)=\left(v_{i}^{k}-\bar{b}^{k}(T)\right) \geq 0
$$

Moreover, if $w^{k}(T) \neq i \forall k \in \mathbb{K}$ then (1) implies that $\gamma_{i}^{k}=0 \forall k$-no item can yield positive surplus to player $i$. Thus, with unit demand, $\mathbf{x}_{i}$ can be no worse for $i$ than any other allocation-including the null allocation-at prices $\mathbf{p}=\overline{\mathbf{b}}(T)$, and condition (iii) is satisfied.

Proof of Lemma 2. Let $\underline{\mathbf{p}}$ denote the minimum Walrasian price vector and suppose that the lemma is false. Note that $\bar{b}^{k}(0) \leq \underline{p}^{k}$. Lemma 1 tells us that (1) always produces a competitive equilibrium outcome. Thus, if (1) does not lead to an allocation supported by $\underline{\mathbf{p}}$, there must be some $\widehat{t}$ such that (i) $\overline{\mathbf{b}}(\hat{t}) \leq \underline{\mathbf{p}}$ and (ii) $\bar{b}^{k}(\widehat{t}+d t)>\underline{p}^{k}$ for some $k$. Denote by $A$ the set of all such $k \in \mathbb{K}$. Since $\bar{b}^{k}(\widehat{t}+d t)$ is the second highest bid for $k$ at time $\widehat{t}+d t$ this implies that, for each $k \in \mathbb{A}$, at least two members of $\square$ have valuations such that $v_{i}^{k}-\bar{b}^{k}(\widehat{t}+d t) \geq v_{i}^{m}-\bar{b}^{m}(\widehat{t}+d t) \forall m \in \mathbb{K} \backslash k$, and, more specifically, such that

(6) $\quad v_{i}^{k}-\bar{b}^{k}(\widehat{t}+d t) \geq v_{i}^{m}-\bar{b}^{m}(\widehat{t}+d t) \forall m \in \mathbb{K} \backslash \mathbb{A}$.

Since (6) is true for at least two $i$ for every $k \in \mathbb{A}$ and players never bid (or hold the provisional winner position) on more than one item simultaneously, it must be true for no fewer than $2|\mathbb{A}|$ members of $\mathbb{0}$.

Now compare $\overline{\mathbf{b}}(\widehat{t}+d t)$ to $\underline{\mathbf{p}}$. We know that $\underline{p}^{k}<\bar{b}^{k}(\widehat{t}+d t) \forall k \in \mathbb{A}$ and that $\underline{p}^{k} \geq$ $\bar{b}^{k}(\widehat{t}+d t) \forall k \in \mathbb{K} \backslash \mathbb{A}$. Thus, there are at least $2|\widehat{A}|$ bidders with $v_{i}^{k}-\underline{p}^{k}>v_{i}^{m}-\underline{p}^{m} \forall m \in$ $\mathbb{K} \backslash \mathbb{A}$ for some $k \in \mathbb{A}$, but only $|\mathbb{A}|$ such items. There must, then, be at least one $k \in \mathbb{A}$ that is in excess demand at prices $\mathbf{p}$, and hence $\underline{\mathbf{p}}$ is not an equilibrium price. The necessary contradiction is thus produced.

\section{REFERENCES}

Anwar, S., R. McMillan, And M. Zheng (2006): "Bidding Behavior in Competing Auctions: Evidence from eBay,” European Economic Review, 50(2), 307-322. 
Ariely, D., A. Ockenfels, And A. E. Roth (2005): "An Experimental Analysis of Ending Rules in Internet Auctions," RAND Journal of Economics, 36(4), 891-908.

BAJARI, P., AND A. HortaçSU (2003): “The Winner's Curse, Reserve Prices and Endogenous Entry: Empirical Insights from eBay Auctions," RAND Journal of Economics, 34(2), 329-355.

(2004): "Economic Insights from Internet Auctions," Journal of Economic Literature, 42(2), 457-486.

ChIANG, K. C. H., AND A. W. P. KUnG (2005): "Bidding dynamics in multi-unit auctions: empirical evidence from online auctions of certificates of deposit," Journal of Financial Intermediation, 14(2), 239-252.

Demange, G., D. Gale, And M. Sotomayor (1986): "Multi-Item Auctions," Journal of Political Economy, 94(4), 869-872.

Ely, J. C., AND T. Hossain (2009): "Sniping and Squatting in Auction Markets," American Economic Journal: Microeconomics, 1(2), 68-94.

Gul, F., And E. Stacchetti (2000): "The English Auction with Differentiated Commodities," Journal of Economic Theory, 92(1), 66-95.

Krishna, V., AND M. Perry (2000): “Efficient Mechanism Design,” Working Paper.

LEONARD, H. B. (1983): "Elicitation of Honest Preferences for the Assignment of Individuals to Positions," Journal of Political Economy, 91(3), 461-479.

Milgrom, P. (2004): Putting Auction Theory to Work. Cambridge University Press, Cambridge, UK.

Ockenfels, A., And A. E. Roth (2006): "Late and Multiple Bidding in Second Price Internet Auctions: Theory and Evidence Concerning Different Rules for Ending an Auction," Games and Economic Behavior, 55(2), 297-320.

Peters, M., AND S. Severinov (2006): "Internet Auctions With Many Traders,” Journal of Economic Theory, 130(1), 220-245.

Roth, A. E., AND A. OCKenfels (2002): "Last-minute bidding and the rules for ending second-price auctions: Evidence from eBay and Amazon auctions on the Internet," American Economic Review, 92(4), 1093-1103.

Shapley, L. S., AND M. Shubik (1971): “The Assignment Game I: The Core," International Journal of Game Theory, 1(1), 111-130. 
Wilcox, R. T. (2000): "Experts and Amateurs: The Role of Experience in Internet Auctions," Marketing Letters, 11(4), 363-374.

Williams, S. R. (1999): "A Characterization of Efficient, Bayesian Incentive Compatible Mechanisms," Economic Theory, 14(1), 155-180.

Zeithammer, R., And C. Adams (2010): “The Sealed-Bid Abstraction in Online Auctions," Marketing Science, 29(6), 964-987. 